\title{
The $r$-matrix structure of the Euler-Calogero-Moser model
}

\author{
E. Billey * \\ J. Avan* \\ O. Babelon *
}

December 1993

\begin{abstract}
We construct the $r$-matrix for the generalization of the Calogero-Moser system introduced by Gibbons and Hermsen. By reduction procedures we obtain the $r$-matrix for the $O(N)$ Euler-Calogero-Moser model and for the standard $A_{N}$ Calogero-Moser model.
\end{abstract}

PAR LPTHE 93-55

*L.P.T.H.E. Université Paris VI (CNRS UA 280), Box 126, Tour 16, $1^{\text {er }}$ étage, 4 place Jussieu, F-75252 PARIS CEDEX 05 


\section{Introduction}

The Calogero-Moser model seems to occupy a particular place in the world of integrable systems. On one hand, its complete solution is known 11, 2, 3, 14 even at the quantum level, but on the other hand, the techniques which have now become standard — in particular the $r$-matrix formalism — did not seem to apply to it.

The beginning of an answer to this problem was given in [5] where the classical $r$-matrix was calculated and found to be a dynamical object, which is a non standard feature. This result was later extended to the elliptic case in [6, 7].

While the general theory of constant classical $r$-matrices is now well developped, this is not so for the dynamical ones and we still are in the phase of producing examples. Dynamical $r$-matrices appear when we consider higher Poisson structures [8, 9]. They also appear in non linear $\sigma$-models [10, 11], or in the theory of Sine Gordon solitons 12]. Finally one should mention an interesting example of dynamical $r$-matrix for the hyperbolic Gaudin model [13].

In this paper we will consider the generalization of the Calogero-Moser model introduced by Gibbons and Hermsen 114. This class of models has recently received some attention in relation with the evolution of energy levels with respect to the parameter $t$ for a perturbated system of Hamiltonian $H=H_{0}+t V$ [15, 16, 17].

Let us introduce a set of dynamical variables $\left(q_{i}, p_{i}\right)_{i=1 \cdots N}$ and $\left(f_{i j}\right)_{i, j=1 \cdots N}$ together with the Poisson brackets

$$
\begin{aligned}
\left\{p_{i}, q_{j}\right\} & =\delta_{i j} \\
\left\{f_{i j}, f_{k l}\right\} & =\delta_{j k} f_{i l}-\delta_{l i} f_{k j}
\end{aligned}
$$

and the Hamiltonian

$$
H=\frac{1}{2} \sum_{i=1}^{N} p_{i}^{2}-\frac{1}{2} \sum_{\substack{i, j=1 \\ i \neq j}}^{N} \frac{f_{i j} f_{j i}}{\left(q_{i}-q_{j}\right)^{2}} .
$$

In order to have a non degenerate Poisson bracket it is assumed that the $\left(f_{i j}\right)_{i, j=1 \cdots N}$ are restricted to a symplectic submanifold of (2).

Notice furthermore that the quantities $\left(f_{i i}\right)_{i=1 \cdots N}$ Poisson commute with the Hamiltonian; we can therefore reduce the flows to the surfaces $\left(f_{i i}=\text { constant }\right)_{i=1 \cdots N}$. These models were shown to be integrable precisely on these surfaces and our purpose is to compute their $r$-matrices.

The plan of the paper is as follows : after a short review on the $r$-matrix formalism in section 2, we find in section 3 the Poisson structure of the Lax operator which implies the integrability property of the model on the manifolds $\left(f_{i i}=\text { constant }\right)_{i=1 \cdots N}$. In section 4 we use the previous result to obtain the $r$-matrix structure for the $O(N)$ Euler-Calogero-Moser model introduced by Wojciechowski [18]. This is achieved by using the mean procedure introduced in [19, 20, 21]. Finally in section 5 we again use the results of section 3 to present a new construction of the $r$-matrix of the standard Calogero-Moser model computed for the first time in [5] and obtained here by Hamiltonian reduction from the model (3).

\section{Dynamical $r$-matrices}

In the Lax representation of a dynamical system, the equations of motion are written in the form

$$
\dot{L}=[L, M]
$$

where $L$ and $M$ take values in a Lie algebra $\mathcal{G}$ with basis $\left\{X_{\mu}\right\}$.

Eq.(11) implies that the quantities $\operatorname{tr}\left(L^{n}\right)$ are conserved. Liouville integrability requires their involution under the Poisson brackets. It was shown in [22] that this property $\left\{\operatorname{tr}\left(L^{m}\right), \operatorname{tr}\left(L^{n}\right)\right\}=0$ is equivalent to the existence of an $r$-matrix.

If we set

$$
L_{1}=L \otimes 1
$$




$$
L_{2}=1 \otimes L
$$

this condition reads

$$
\left\{L_{1}, L_{2}\right\}=\left[r_{12}, L_{1}\right]-\left[r_{21}, L_{2}\right]
$$

where

$$
r_{12}=\sum r_{\mu \nu} X_{\mu} \otimes X_{\nu} \quad \text { and } \quad r_{21}=\sum r_{\mu \nu} X_{\nu} \otimes X_{\mu} .
$$

In general the $r$-matrix is a dynamical object and possesses no special symmetry. Let us recall here some of its properties.

1. The Lax operator $L$ together with the $r$-matrix completely describe the dynamical system: if we choose $\frac{1}{n} \operatorname{tr}\left(L^{n}\right)$ as a Hamiltonian, the equations of motion take the Lax form with $M=\operatorname{tr}_{2}\left(r_{12} L_{2}^{n-1}\right)$.

2. The left-hand side of eq.(5) being a Poisson bracket, the Jacobi identity implies a constraint on $r$. It is easy to check that it takes the form

$$
\begin{gathered}
{\left[L_{1},\left[r_{12}, r_{13}\right]+\left[r_{12}, r_{23}\right]+\left[r_{32}, r_{13}\right]+\left\{L_{2}, r_{13}\right\}-\left\{L_{3}, r_{12}\right\}\right]} \\
+ \text { cyclic permutations }=0
\end{gathered}
$$

It is interesting to note that the display of indices in (6) is not the one which appears in the usual Yang-Baxter equation. We recover it when the $r$-matrix is antisymmetric.

3. Since the existence of an $r$-matrix is equivalent to the involution property of the eigenvalues of $L$, the form of eq.(5) is not modified by a conjugation of the Lax matrix. Indeed if $L^{\prime}=g^{-1} L g$ then

$$
\left\{L_{1}^{\prime}, L_{2}^{\prime}\right\}=\left[r_{12}^{\prime}, L_{1}^{\prime}\right]-\left[r_{21}^{\prime}, L_{2}^{\prime}\right]
$$

with

$$
r_{12}^{\prime}=g_{1}^{-1} g_{2}^{-1}\left(r_{12}-\left\{g_{1}, L_{2}\right\} g_{1}^{-1}+\frac{1}{2}\left[u_{12}, L_{2}\right]\right) g_{1} g_{2}
$$

where $u_{12}=\left\{g_{1}, g_{2}\right\} g_{1}^{-1} g_{2}^{-1}$.

4. Finally we recall that the $r$-matrix of a given Lax matrix is not unique. In particular if we introduce a symmetric matrix $\tau_{12}=\tau_{21}$ of $\mathcal{G} \otimes \mathcal{G}$, the change

$$
r_{12} \longrightarrow r_{12}+\left[\tau_{12}, L_{2}\right]
$$

does not affect the equation (5).

\section{The Poisson structure for the $s l(N)$ model}

The Lax matrix of the system (3) is

$$
L(\lambda)=\sum_{i=1}^{N}\left(p_{i}-\frac{f_{i i}}{\lambda}\right) e_{i i}-\sum_{\substack{i, j=1 \\ i \neq j}}^{N}\left(\frac{1}{q_{i}-q_{j}}+\frac{1}{\lambda}\right) f_{i j} e_{i j} .
$$

where $\left(e_{i j}\right)_{k l}=\delta_{i k} \delta_{j l}$.

The Poisson brackets of the elements of the Lax matrix (9) can be recast in the form

$$
\left\{L_{1}(\lambda), L_{2}(\mu)\right\}=\left[r_{12}(\lambda, \mu), L_{1}(\lambda)\right]-\left[r_{21}(\mu, \lambda), L_{2}(\mu)\right]-\sum_{\substack{i, j=1 \\ i \neq j}}^{N} \frac{f_{i i}-f_{j j}}{\left(q_{i}-q_{j}\right)^{2}} e_{i j} \otimes e_{j i}
$$

where

$$
r_{12}(\lambda, \mu)=-\frac{\mathcal{C}}{\lambda-\mu}-\sum_{\substack{i, j=1 \\ i \neq j}}^{N} \frac{1}{q_{i}-q_{j}} e_{i j} \otimes e_{j i}
$$


and $\mathcal{C}$ is the Casimir element of $\operatorname{sl}(N)$

$$
\mathcal{C}=\sum_{i, j=1}^{N} e_{i j} \otimes e_{j i}
$$

Some comments are in order:

1. Eq.(10) holds for the non reduced dynamical system (3) and it is known that $\operatorname{tr} L^{n}(\lambda)$ are not in involution for this system. This fact is responsible for the additional term in eq. (10) compared to eq.(5). Indeed we have

$$
\left\{\operatorname{tr} L^{n}(\lambda), \operatorname{tr} L^{m}(\mu)\right\}=n m \sum_{\substack{i, j=1 \\ i \neq j}}^{N} \frac{f_{i i}-f_{j j}}{\left(q_{i}-q_{j}\right)^{2}}\left[L^{n-1}(\lambda)\right]_{i j}\left[L^{m-1}(\mu)\right]_{j i} .
$$

2. The constraints $f_{i i}$ generate on $L(\lambda)$ a conjugation by a diagonal matrix. Hence $\operatorname{tr} L^{n}(\lambda)$ Poisson commute with $f_{i i}$. It follows that one can compute their reduced Poisson bracket on the manifold $\left(f_{i i}=\text { constant }\right)_{i=1 \cdots N}$. This amounts to setting $f_{i i}=$ constant in eq.(10) and therefore $\operatorname{tr} L^{n}(\lambda)$ are in involution.

3. Since the quantities $\operatorname{tr} L^{n}(\lambda)$ commute on the reduced phase space, $L(\lambda)$ has an $r$-matrix. However its explicit computation requires some care since $L(\lambda)$ is not a function on the reduced phase space.

In the following two sections we give two examples of reductions where we can explicitely obtain the $r$-matrix, starting from the initial structure (10).

\section{The $r$-matrix for the $O(N)$ model}

We now consider the following model, introduced by Wojciechowski in [18]. The dynamical variables are $\left(q_{i}, p_{i}\right)_{i=1 \cdots N}$ and antisymmetric $\left(h_{i j}=-h_{j i}\right)_{i, j=1 \cdots N}$. The Poisson bracket is now:

$$
\begin{aligned}
\left\{p_{i}, q_{j}\right\} & =\delta_{i j} \\
\left\{h_{i j}, h_{k l}\right\} & =\frac{1}{2}\left(\delta_{i l} h_{j k}+\delta_{k i} h_{l j}+\delta_{j k} h_{i l}+\delta_{l j} h_{k i}\right) .
\end{aligned}
$$

The Hamiltonian of the system reads

$$
H=\frac{1}{2} \sum_{i=1}^{N} p_{i}^{2}+\frac{1}{2} \sum_{\substack{i, j=1 \\ i \neq j}}^{N} \frac{h_{i j}^{2}}{\left(q_{i}-q_{j}\right)^{2}} .
$$

As shown in [18] the equations of motion can be written in the form

$$
\dot{L}(\lambda)=[L(\lambda), M(\lambda)]
$$

where the Lax pair $(L, M)$ is given by

$$
\begin{aligned}
L(\lambda) & =\sum_{i=1}^{N} p_{i} e_{i i}-\sum_{\substack{i, j=1 \\
i \neq j}}^{N}\left(\frac{1}{q_{i}-q_{j}}+\frac{1}{\lambda}\right) h_{i j} e_{i j} \\
M(\lambda) & =\sum_{\substack{i, j=1 \\
i \neq j}}^{N} \frac{h_{i j}}{\left(q_{i}-q_{j}\right)^{2}} e_{i j}
\end{aligned}
$$

and the Hamiltonian is $H=\frac{1}{2} \int \frac{d \lambda}{2 i \pi \lambda} \operatorname{tr} L(\lambda)^{2}$.

In [18] this system was proved to have Poisson commuting Hamiltonians $\operatorname{tr} L^{n}(\lambda)$. It follows that this model admits an $r$-matrix, which we now calculate. 
First of all we remark that the Lax matrix of the $O(N)$ model is obtained from the Lax matrix of the $s l(N)$ model as

$$
L^{O(N)}(\lambda)=\frac{1}{2}(1-\sigma) L^{s l(N)}(\lambda)
$$

where $\sigma$ is the following involutive automorphism of the loop algebra:

$$
\sigma: \lambda^{n} e_{i j} \longrightarrow-(-\lambda)^{n} e_{j i} \text {. }
$$

In this average procedure

$$
h_{i j}=\frac{1}{2}\left(f_{i j}-f_{j i}\right)
$$

and the Poisson structure (2) of the $\operatorname{sl}(N)$ model becomes the structure (13).

It follows immediately that

$$
\begin{aligned}
\left\{L_{1}^{O(N)}(\lambda), L_{2}^{O(N)}(\mu)\right\}= & \frac{1}{4}(1-\sigma \otimes 1-1 \otimes \sigma+\sigma \otimes \sigma) \cdot \\
& \left(\left[r_{12}(\lambda, \mu), L_{1}^{s l(N)}(\lambda)\right]-\left[r_{21}(\mu, \lambda), L_{2}^{s l(N)}(\mu)\right]-\sum_{\substack{i, j=1 \\
i \neq j}}^{N} \frac{f_{i i}-f_{j j}}{\left(q_{i}-q_{j}\right)^{2}} e_{i j} \otimes e_{j i}\right) .
\end{aligned}
$$

We now remark that

$$
(1-\sigma \otimes 1-1 \otimes \sigma+\sigma \otimes \sigma) \sum_{\substack{i, j=1 \\ i \neq j}}^{N} \frac{f_{i i}-f_{j j}}{\left(q_{i}-q_{j}\right)^{2}} e_{i j} \otimes e_{j i}=0
$$

Moreover since

$$
\sigma \otimes \sigma r_{12}(\lambda, \mu)=-r_{12}(\lambda, \mu)
$$

we finally get an explicit $r$-matrix structure for $L^{O(N)}(\lambda)$ :

$$
\left\{L_{1}^{O(N)}(\lambda), L_{2}^{O(N)}(\mu)\right\}=\left[r_{12}^{O(N)}(\lambda, \mu), L_{1}^{O(N)}(\lambda)\right]-\left[r_{21}^{O(N)}(\mu, \lambda), L_{2}^{O(N)}(\mu)\right]
$$

with

$$
r_{12}^{O(N)}(\lambda, \mu)=\frac{1}{2}(1+\sigma \otimes 1) r_{12}^{s l(N)}(\lambda, \mu)
$$

or explicitely

$$
\begin{aligned}
r_{12}^{O(N)}(\lambda, \mu)=-\frac{\lambda}{\lambda^{2}-\mu^{2}} \sum_{i=1}^{N} e_{i i} \otimes e_{i i} & -\frac{1}{2} \sum_{\substack{i, j=1 \\
i \neq j}}^{N}\left(\frac{1}{q_{i}-q_{j}}+\frac{1}{\lambda+\mu}\right) e_{i j} \otimes e_{i j} \\
& -\frac{1}{2} \sum_{\substack{i, j=1 \\
i \neq j}}^{N}\left(\frac{1}{q_{i}-q_{j}}+\frac{1}{\lambda-\mu}\right) e_{i j} \otimes e_{j i} .
\end{aligned}
$$

This is an example of application of the well known mean procedure 19, 20, 21.

It is interesting to see how the Jacobi identity (6) is fulfilled for this dynamical $r$-matrix (24). We find that

$$
\left[r_{12}, r_{13}\right]+\left[r_{12}, r_{23}\right]+\left[r_{32}, r_{13}\right]=\frac{1}{2} \sum_{\substack{i, j=1 \\ i \neq j}}^{N} \frac{1}{\left(q_{i}-q_{j}\right)^{2}} e_{i j} \otimes\left[\left(e_{i j}+e_{j i}\right) \otimes\left(e_{i i}-e_{j j}\right)-\left(e_{i i}-e_{j j}\right) \otimes\left(e_{i j}+e_{j i}\right)\right] .
$$

It is then easy to check that

$$
\left[r_{12}, r_{13}\right]+\left[r_{12}, r_{23}\right]+\left[r_{32}, r_{13}\right]+\left\{L_{2}, r_{13}\right\}-\left\{L_{3}, r_{12}\right\}=0 .
$$

The remarkable feature of this equation is the vanishing of its right-hand side. This is in contrast with other cases [6, 13] where eq.25) has a non vanishing right-hand side, albeit of a special form. 


\section{A new construction of the $r$-matrix for the Calogero model}

We now show how the dynamical $r$-matrix for the usual Calogero model [5, 6, 7] stems from eq.(10) through a procedure of Hamiltonian reduction.

Let us restrict oneselves to the symplectic manifold

$$
f_{i j}=\xi_{i} \eta_{j}
$$

with $\left\{\xi_{i}, \xi_{j}\right\}=0,\left\{\eta_{i}, \eta_{j}\right\}=0,\left\{\xi_{i}, \eta_{j}\right\}=-\delta_{i j}$. The Poisson brackets of $f_{i j}$ are indeed given by (2).

On this manifold we have a symplectic action of an Abelian Lie group

$$
\xi_{i} \longrightarrow \lambda_{i} \xi_{i}, \quad \eta_{i} \longrightarrow \lambda_{i}^{-1} \eta_{i} .
$$

The Hamiltonian (3) is invariant under this action and we can apply the method of Hamiltonian reduction. The infinitesimal generator of this action is

$$
H_{\epsilon}=\sum_{i=1}^{N} \epsilon_{i} f_{i i}
$$

The reduction is performed by first fixing the momentum. We choose:

$$
f_{i i}=\alpha .
$$

The isotropy group $G_{\alpha}$ of $\alpha$ is the whole group itself since it is Abelian and we still have to quotient out the action of this group. At the end of the procedure, the $2 N$ degrees of freedom $\left(\xi_{i}, \eta_{i}\right)_{i=1 \cdots N}$ are eliminated, leaving as reduced Hamiltonian (3) the Hamiltonian of the usual Calogero-Moser model

$$
H_{\text {Cal }}=\frac{1}{2} \sum_{i=1}^{N} p_{i}^{2}-\frac{1}{2} \alpha^{2} \sum_{\substack{i, j=1 \\ i \neq j}}^{N} \frac{1}{\left(q_{i}-q_{j}\right)^{2}}
$$

In order to perform the reduction at the level of the Lax matrix we remark that the group acts on $L$ as conjugation by the matrix $\operatorname{diag}\left(\lambda_{i}\right)_{i=1 \cdots N}$. We now observe that the matrix

$$
L_{\text {Cal }}=\operatorname{diag}\left(\xi_{i}^{-1}\right) L \operatorname{diag}\left(\xi_{i}\right)
$$

is invariant under $G_{\alpha}$ and we can therefore compute the Poisson brackets of its matrix elements safely.

$$
L_{\text {Cal }}=\sum_{i=1}^{N}\left(p_{i}-\frac{\alpha}{\lambda}\right) e_{i i}-\alpha \sum_{\substack{i, j=1 \\ i \neq j}}^{N}\left(\frac{1}{q_{i}-q_{j}}+\frac{1}{\lambda}\right) e_{i j}
$$

The $r$-matrix of this $L$ matrix is now obtained from eq.(10) and (11) by applying the conjugation formula (7).

When setting $f_{i i}=\alpha$ the extra term in eq. (10) vanishes, leaving us with the $r$-matrix

$$
r_{12}^{\mathrm{Cal}}(\lambda, \mu)=r_{12}(\lambda, \mu)-\frac{1}{\mu} \sum_{i=1}^{N} e_{i i} \otimes e_{i i}+\sum_{\substack{i, j=1 \\ i \neq j}}^{N}\left(\frac{1}{q_{i}-q_{j}}-\frac{1}{\mu}\right) e_{i i} \otimes e_{j i}
$$

which gives

$$
r_{12}^{\mathrm{Cal}}(\lambda, \mu)=-\frac{\lambda}{\mu(\lambda-\mu)} \sum_{i=1}^{N} e_{i i} \otimes e_{i i}-\sum_{\substack{i, j=1 \\ i \neq j}}^{N}\left(\frac{1}{q_{i}-q_{j}}+\frac{1}{\lambda-\mu}\right) e_{i j} \otimes e_{j i}+\sum_{\substack{i, j=1 \\ i \neq j}}^{N}\left(\frac{1}{q_{i}-q_{j}}-\frac{1}{\mu}\right) e_{i i} \otimes e_{j i} .
$$

This $r$-matrix does not coincide with the $r$-matrix obtained in [5, 6, 7], however it is easily related to it by a transformation (8) with $\tau_{12}=\frac{1}{2} \sum_{i=1}^{N} e_{i i} \otimes e_{i i}$. 


\section{Conclusion}

The class of models introduced by Gibbons and Hermsen [14 turns out to be a particularly interesting generalization of the Calogero model. In particular their $r$-matrix structure is rather elegant and is general enough to allow the construction of a host of new models by various reduction procedures. Generalization to trigonometric and elliptic potentials is possible and will be developped further.

Acknowledgements It is a pleasure to thank Denis Bernard for discussions.

\section{References}

[1] F. Calogero, J. Math. Phys. 12 (1971) 419.

[2] F.Dunkl, Trans. Amer. Math. Soc. 311 (1989) 167.

[3] A. Polychronakos, Phys. Rev. Lett. 69 (1992) 703.

[4] L. Brink, T.H. Hanssen and M.A. Vasiliev, Phys. Lett. B 286 (1992) 109.

[5] J. Avan and M. Talon, Phys. Lett. B 303 (1993) 33.

[6] E.K. Sklyanin, preprint LPTHE-93-42; hep-th/9308060.

[7] H.W. Braden and T. Suzuki, preprint University of Edinburgh-92/93-03; hep-th/9309033.

[8] M. Adler, Inv. Math. 50 (1979) 219.

[9] E.K. Sklyanin, Funct. Anal. Appl. 16 (1982) 263.

[10] M. Bordemann, M. Forger, J. Laartz and U. Schlager, Comm. Math. Phys. 152 (1993) 167.

[11] J-M. Maillet, Phys. Lett. B 162 (1985) 137.

[12] O. Babelon and D. Bernard, Phys. Lett. B 317 (1993) 363; hep-th/9309154.

[13] J.C. Eilbeck, V.Z. Enol'skii, V.B. Kuznetsov and A.V. Tsiganov, preprint hep-th/9306155.

[14] J. Gibbons and T. Hermsen, Physica 11D (1984) 337.

[15] P. Pechukas, Phys. Rev. Lett. 51 (1983) 943.

[16] T. Yukawa, Phys. Rev. Lett. 54 (1985) 1883.

[17] K. Nakamura and M. Lakshmanan, Phys. Rev. Lett. 57 (1986) 1661.

[18] S. Wojciechowski, Phys. Lett. 111A (1985) 101.

[19] A.V. Mikhailov, JETP Lett. 30 (1979) 443.

[20] L.D. Faddeev and N.Yu. Reshetikhin, Theor. Math. Phys. 56 (1993) 323.

[21] J. Avan and M. Talon, Nucl. Phys. B 352 (1991) 215.

[22] O. Babelon and C-M. Viallet, Phys. Lett. B 237 (1990) 411. 